\title{
Effect of vitamin $E$ in nonalcoholic fatty liver disease with metabolic syndrome: A propensity score-matched cohort study
}

\author{
Gi Hyun Kim', Jung Wha Chung', Jong Ho Lee', Kyeong Sam Ok', Eun Sun Jang ${ }^{1,2}$, Jaihwan Kim¹, Cheol Min Shin', \\ Young Soo Park ${ }^{1,2}$, Jin-Hyeok Hwang ${ }^{1,2}$, Sook-Hyang Jeong ${ }^{1,2}$, Nayoung Kim ${ }^{1,2}$, Dong Ho Lee, ${ }^{1,2}$ and Jin-Wook Kim ${ }^{1,2}$ \\ 'Department of Medicine, Seoul National University Bundang Hospital, Seongnam; ${ }^{2}$ Department of Internal Medicine, Seoul National \\ University College of Medicine, Seoul, Korea
}

Background/Aims: Vitamin E improves the biochemical profiles and liver histology in nonalcoholic steatohepatitis, but the role of vitamin $E$ is not clearly defined in the management of nonalcoholic fatty liver disease (NAFLD) which includes both simple steatosis and steatohepatitis. Co-morbid metabolic syndrome increases the probability of steatohepatitis in NAFLD. In this study, we aimed to determine the short-term effects of vitamin E and off-treatment durability of response in a propensity-score matched cohort of NAFLD patients with metabolic syndrome.

Methods: A retrospective cohort was constructed by retrieving 526 consecutive NAFLD patients from the electronic medical record data warehouse of a tertiary referral hospital in South Korea. Among them, 335 patients (63.7\%) had metabolic syndrome and were eligible for vitamin $E$ therapy. In order to assess the effect of vitamin $E$, propensity score matching was used by matching covariates between control patients $(n=250)$ and patients who received vitamin $E(n=85)$.

Results: The PS-matched vitamin E group ( $n=58)$ and control group $(n=58)$ exhibited similar baseline metabolic profiles. After 6 months of vitamin E therapy, the mean ALT levels decreased significantly compared to PS-matched control $(P<0.01)$. The changes in metabolic profiles (body weight, lipid and glucose levels) did not differ between control and vitamin $E$ groups during the study period.

Conclusions: Short-term vitamin E treatment significantly reduces ALT levels in NAFLD patients with metabolic syndrome, but metabolic profiles are not affected by vitamin E. (Clin Mol Hepatol 2015;21:379-386)

Keywords: Nonalcoholic fatty liver disease; Vitamin E; Metabolic syndrome; Propensity score

\section{INTRODUCTION}

Nonalcoholic fatty liver disease (NAFLD) is one of the most common types of chronic liver diseases worldwide. ${ }^{1,2}$ NAFLD en- compasses a range of histologic abnormalities from simple steatosis to more severe nonalcoholic steatohepatitis (NASH) and liver cirrhosis. ${ }^{3,4}$ NAFLD is associated with increased risks of cardiovascular and liver-related mortality, ${ }^{5,6}$ especially in steatohepatitis. ${ }^{3}$

\footnotetext{
Abbreviations:

ALT, alanine transaminase; APRI, AST-to-platelet ratio index; AST, aspartate transaminase; BMI, body mass index; EMR, electronic medical record; GGT, gamma-glutamyltransferase; HOMA-IR, homeostasis model assessment of insulin resistance; NAFLD, nonalcoholic fatty liver disease; NASH, nonalcoholic steatohepatitis; PS, propensity score
}

\section{Corresponding author : Jin-Wook Kim}

Department of Medicine, Seoul National University Bundang Hospital,

82 Gumi-ro 173 beon-gil, Bundang-gu, Seongnam 13620, Korea

Tel: +82-31-787-7013, Fax: +82-31-787-4051

E-mail: kimjw@snubh.org 
Currently, the main strategy for the management of NAFLD is the control of metabolic comorbidities by life style modification? Various medications have been tried in the treatment of NAFLD without success, with the exception of pioglitazone and vitamin E. ' Oxidative stress has been implicated as a major mechanism of disease progression in NAFLD, and vitamin $E$, a potent antioxidant, may suppress hepatic inflammation by inactivating free radicals and suppressing lipid peroxidation. ${ }^{9-11}$ Randomized controlled trials showed that vitamin $\mathrm{E}$ reduced serum aminotransferase levels and improved lobular inflammation in NASH. 12,13 Based on these findings, current guidelines recommend vitamin $E$ for NAFLD patients with steatohepatitis ${ }^{7,14}$. However, liver biopsy is necessary to confirm the presence of steatohepatitis in NAFLD, which is a major obstacle to vitamin E therapy in NAFLD patients. Since presence of metabolic syndrome carried a high risk of steatohepatitis in NAFLD, ${ }^{15-17}$ it can be speculated that NAFLD patients with metabolic syndrome may benefit from vitamin $\mathrm{E}$ therapy. However, the effect of vitamin $E$ in this group has not been studied yet.

The propensity score (PS) is the conditional probability of receiving a certain treatment according to an individual's covariates. ${ }^{18}$ PS matching is increasingly being used to balance covariates between 2 groups, reducing the bias in observational studies. ${ }^{19,20}$ In this study, therefore, we sought to determine the short-term effect of vitamin $E$ and its durability in NAFLD patients with metabolic syndrome by comparison with PS-matched control.

\section{PATIENTS AND METHODS}

\section{Study population}

This was a retrospective cohort study conducted in a tertiary referral hospital (Seoul National University Bundang Hospital) in South Korea. A NAFLD cohort was retrieved from a structured chronic liver disease database that has been maintained since 2003 as part of BESTCare, the electronic medical record (EMR) system developed by our hospital. ${ }^{21}$ All consecutive NAFLD patients $>18$ years old who visited our hospital between May 2003 and December 2013, had elevated baseline alanine aminotransferase (ALT, >40 IU/L) and were followed up for at least 6months were retrieved. The diagnosis of NAFLD was made according to the presence of steatosis on ultrasonography and exclusion of the secondary hepatic fat accumulation. ${ }^{22}$ The presence of metabolic syndrome was determined according to the NCEP ATP III crite- ria ${ }_{1}^{23}$ modified by International Diabetes Federation: ${ }^{24}$ the presence of three or more of 1) abdominal obesity: waist circumference $>90$ $\mathrm{cm}$ in men and $>80 \mathrm{~cm}$ in women; 2) hypertriglyceridemia: serum TG level $\geq 150 \mathrm{mg} / \mathrm{dL}$ or drug treatment for elevated TG; 3) low HDL-cholesterol: $<40 \mathrm{mg} / \mathrm{dL}$ in men and $<50 \mathrm{mg} / \mathrm{dL}$ in women or drug treatment for low HDL-C; 4) high blood pressure: SBP $\geq 130$ $\mathrm{mmHg}$ and/or $\mathrm{DBP} \geq 85 \mathrm{mmHg}$ or drug treatment for elevated blood pressure; 5) high fasting glucose (FBS): serum glucose level $\geq 100 \mathrm{mg} / \mathrm{dL}$ or being under treatment for diabetes. If waist circumference data were not available, central obesity was determined by body mass index (BMI) with the cut-off of 25 on the basis of the Asia-Pacific working party guideline. ${ }^{25}$ The exclusion criteria were: 1) other causes of steatosis (i.e., excessive alcohol consumption; steatogenic medications such as amiodarone, methotrexate, tamoxifen, and corticosteroids; parenteral nutrition and pregnancy); 2) other causes of ALT elevation (viral hepatitis, autoimmune liver diseases, toxic hepatitis, hemochromatosis, Wilson disease); 3) comorbidity (generalized inflammatory diseases or advanced malignant diseases); 4) patients on multi-vitamins before visiting outpatient clinic. Excessive alcohol consumption was defined as more than $20 \mathrm{~g}$ per day in women, and more than $30 \mathrm{~g}$ per day in men for at least 3 consecutive months. Drug and alcohol history was recorded for all patients and anthropometric data, such as height and body weight, were measured through the outpatient clinic. Serum aspartate transaminase (AST), ALT, gamma-glutamyltransferase (GGT), total bilirubin, cholesterol, triglycerides, high-density lipoprotein cholesterol, and low-density lipoprotein cholesterol levels were initially measured and re-assessed every 3 months. Serum insulin level was also measured to determine insulin resistance. The homeostasis model assessment of insulin resistance (HOMA-IR) was calculated according to the following formula: fasting plasma glucose (mg/ $\mathrm{dL}) \times$ fasting insulin $(\mu \mathrm{U} / \mathrm{mL}) / 405$. The NAFLD fibrosis score and AST-to-platelet ratio index (APRI) score were calculated as previously reported. ${ }^{26,27}$

All NAFLD patients with metabolic syndrome were recommended to follow caloric restriction and increased physical activity during the study period. When ALT levels were not improved after 6-12 months of life style modification, Vitamin $\mathrm{E}$ or hepatotonic agents were considered. The final decision on prescription rested on the discretion of 3 attending physicians (ESJ, SHJ, and JWK).

An improvement in ALT level was defined as a decrease to $\leq$ $40 \mathrm{U} / \mathrm{L}$ and by $\geq 30 \%$ of the baseline. ${ }^{13}$ The institutional review board approved this study (B-1406-256-113). 


\section{PS matching}

We used PS matching between vitamin $\mathrm{E}$ and control groups to adjust the baseline characteristics of NAFLD. The values for matching parameters were chosen at least 6 months after lifestyle modification. The variables for matching were age, sex, BMI, ALT, AST, diabetes mellitus, and dyslipidemia. Diabetes mellitus was defined as fasting glucose level $\geq 126 \mathrm{mg} / \mathrm{dL}$. Dyslipidemia was defined as fasting cholesterol level $\geq 220 \mathrm{mg} / \mathrm{dL}$. PS were estimated non-parametrically using the "Matchlt" function in the $\mathrm{R}$ software package. Matching was performed by the nearest neighbor matching method using a caliper width of 0.1 multiplied by the standard deviation for linearly transformed propensity scores (i.e., logit-transformation).

\section{Statistical analysis}

All statistical analyses were performed using SPSS version 21.0 (SPSS, Chicago, IL, USA). Anthropometric, biochemical, and histological variables were categorized as continuous or categorical variables. Continuous and categorical variables were compared between groups using Student $t$-test and the $\chi^{2}$ test, respectively. Logistic regression analysis was performed to assess the effects of vitamin E treatment after adjusting for confounders and identifying predictors of vitamin $\mathrm{E}$ response. All statistical tests were two-sided. The level of significance was set at $P<0.05$.

\section{RESULTS}

\section{Characteristics of the study population}

A total of 2,828 patients with ultrasonographically diagnosed fatty liver were retrieved from the BESTCare database. Among them, 2,302 patients were excluded because of low initial ALT levels (<40 IU/L), short follow-up duration ( $<6$ months) or other exclusion conditions which may affect transaminase levels. Among the remaining 526 NAFLD patients, 335 (63.7\%) had metabolic syndrome and were eligible for vitamin $E$ therapy. Two hundred fifty patients were followed without vitamin $E_{\text {, and }} 85$ patients received vitamin $\mathrm{E}$ during the study period. Vitamin $\mathrm{E}$ ( $\alpha$-tocopherol) was prescribed at an average dosage of $883 \mathrm{IU} /$ day. The mean duration of treatment was 182 days. After exclusion of 3 patients with poor compliance, 82 vitamin E-treated patients and 250 control patients were matched for PS. Finally, 58 matched patients from each group were selected for analysis (Fig. 1).

Table 1 shows comparison of baseline characteristics between the two groups. Before PS matching, the vitamin E group had younger patients with higher BMI, higher prevalence of diabetes, higher initial levels of ALT, AST, GGT, and APRI score. After PS

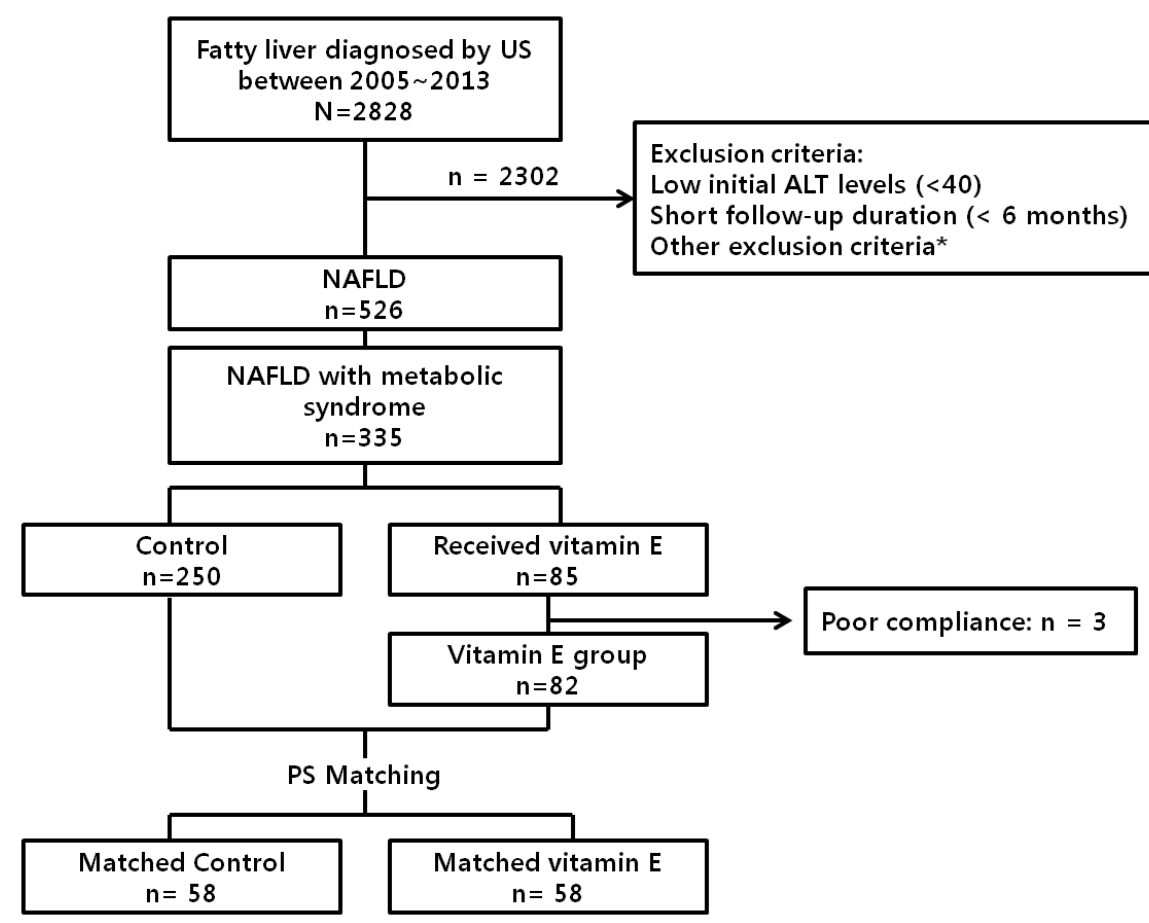

Figure 1. Flow chart of study algorithm. *Excessive alcohol consumption, steatogenic drug, pregnancy, viral hepatitis, autoimmune liver disease, toxic hepatitis, genetic liver disease, gallstone, thyroid disease, heart failure, malignancies, on vitamins before enrollment. Excessive alcohol consumption was defined as more than $20 \mathrm{~g}$ per day in women, and more than 30 $g$ per day in men, for at least 3 consecutive months. Autoimmune liver disease included autoimmune hepatitis and primary biliary cirrhosis. PS, propensity score; US, ultrasonography; ALT, alanine aminotransferase; NAFLD, nonalcoholic fatty liver disease. 
Table 1. Baseline characteristics of the study subjects

\begin{tabular}{|c|c|c|c|c|c|c|}
\hline & \multicolumn{3}{|c|}{ Total population } & \multicolumn{3}{|c|}{ Propensity score-matched population } \\
\hline & $\begin{array}{l}\text { Control } \\
(n=250)\end{array}$ & $\begin{array}{l}\text { Vitamin } E \\
(n=82)\end{array}$ & $P$-value & $\begin{array}{l}\text { Control } \\
(n=58)\end{array}$ & $\begin{array}{l}\text { Vitamin E } \\
(n=58)\end{array}$ & $P$-value \\
\hline Age (years) & $55 \pm 12$ & $51 \pm 15$ & 0.03 & $54 \pm 14$ & $53 \pm 15$ & 0.74 \\
\hline Male sex (\%) & 69 & 73.2 & 0.49 & 66 & 69 & 0.69 \\
\hline Body weight (kg) & $77 \pm 14$ & $81 \pm 14$ & 0.04 & $78 \pm 14$ & $79 \pm 13$ & 0.59 \\
\hline BMI & $27.5 \pm 3.2$ & $28.5 \pm 3.4$ & 0.02 & $27.9 \pm 3.4$ & $28.2 \pm 3.3$ & 0.57 \\
\hline Waist circumference $(\mathrm{cm})$ & $93 \pm 7$ & $96 \pm 6$ & 0.23 & $92 \pm 6$ & $94 \pm 5$ & 0.56 \\
\hline Hypertension (\%) & 53 & 51 & 0.76 & 53 & 50 & 0.71 \\
\hline HOMA-IR & $4.9 \pm 2.8$ & $5.4 \pm 5.6$ & 0.53 & $5.5 \pm 3.0$ & $5.7 \pm 6.8$ & 0.92 \\
\hline Diabetes mellitus (\%) & 43 & 59 & 0.02 & 53 & 59 & 0.58 \\
\hline Dyslipidemia (\%) & 75 & 63 & 0.05 & 66 & 62 & 0.70 \\
\hline $\operatorname{ALT}(\mathrm{U} / \mathrm{L})$ & $61 \pm 38$ & $118 \pm 52$ & 0.01 & $99 \pm 55$ & $106 \pm 52$ & 0.51 \\
\hline AST (U/L) & $39 \pm 17$ & $65 \pm 24$ & 0.01 & $59 \pm 22$ & $58 \pm 23$ & 0.92 \\
\hline GGT (U/L) & $68 \pm 53$ & $87 \pm 45$ & 0.01 & $88 \pm 74$ & $79 \pm 41$ & 0.40 \\
\hline Bilirubin (mg/dL) & $1.0 \pm 0.4$ & $0.9 \pm 0.3$ & 0.24 & $0.6 \pm 0.4$ & $0.6 \pm 0.4$ & 0.98 \\
\hline NAFLD fibrosis score ${ }^{\dagger}$ & $-1.85 \pm 1.20$ & $-1.75 \pm 1.37$ & 0.57 & $-1.80 \pm 1.33$ & $-1.71 \pm 1.39$ & 0.74 \\
\hline APRI score & $0.43 \pm 0.20$ & $0.74 \pm 0.32$ & 0.01 & $0.61 \pm 0.24$ & $0.65 \pm 0.27$ & 0.42 \\
\hline
\end{tabular}

Values are presented as mean \pm standard deviation or percentages.

ALT, alanine aminotransferase; AST, aspartate aminotransferase; GGT, gamma-glutamyltransferase; NAFLD, nonalcoholic fatty liver disease; APRI, AST-toplatelet ratio index; BMI, body mass index; IFG, impaired fasting glucose.

"Glucose $(\mathrm{mg} / \mathrm{dL}) \times$ insulin $(\mathrm{mU} / \mathrm{L}) / 405$.

${ }^{\dagger}$ NAFLD fibrosis score $=-1.675+0.037 \times$ age $($ years $)+0.094 \times \mathrm{BMI}\left(\mathrm{kg} / \mathrm{m}^{2}\right)+1.13 \times \mathrm{IFG} /$ diabetes $($ yes $=1, \mathrm{no}=0)+0.99 \times$ AST/ALT ratio- $0.013 \times$ platelet $\left(\times 10^{9} / \mathrm{L}\right)$ - 0.66xalbumin ( $\mathrm{g} / \mathrm{dL})$.

${ }^{\ddagger}$ AST $(\mathrm{IU} / \mathrm{L}) /\left(40 \times\right.$ platelet $\left.\left(\times 10^{9} / \mathrm{L}\right)\right) \times 100$.
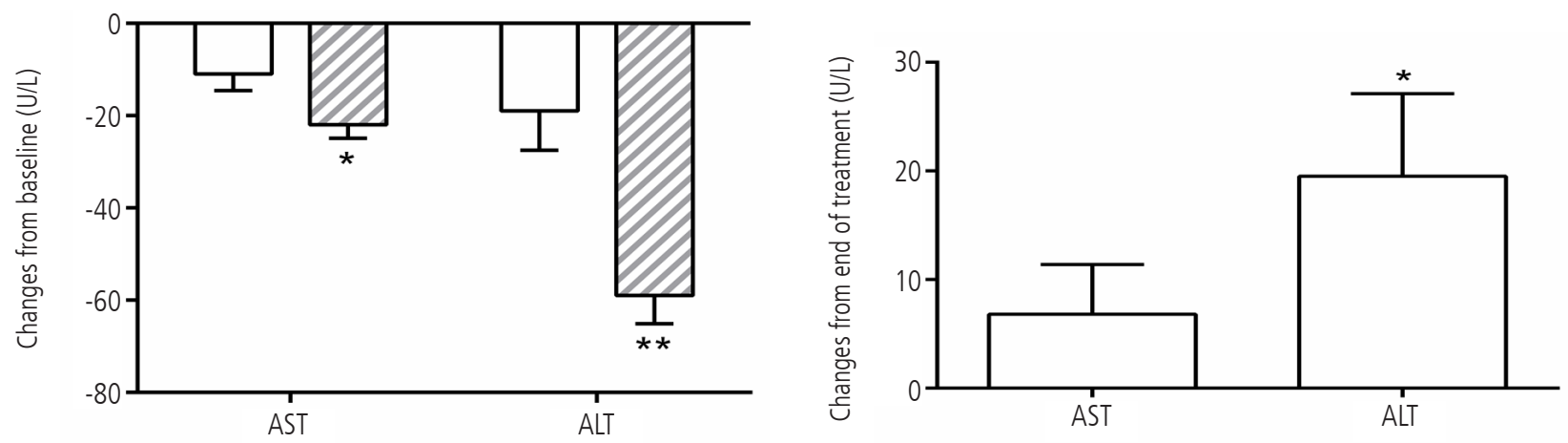

\section{A $\square$ Control $\square$ Vitamin E}

B

Figure 2. Changes of serum aminotransferase levels during and after vitamin E treatment in NAFLD patients with metabolic syndrome. Mean values and standard errors of mean are shown for changes in AST and ALT levels relative to baseline over 6 months of life style modification alone (control) or life style modification plus vitamin $E$ therapy (A), and over 6 months of follow-up after cessation of vitamin $E$ therapy (B). NAFLD, nonalcoholic fatty liver disease; AST, aspartate transaminase; ALT, alanine transaminase. ${ }^{*} P<0.05 ;{ }^{* *} P<0.01$.

matching, however, those characteristics became comparable between the two groups.

\section{Effect of vitamin E on transaminase levels in NAFLD patients with metabolic syndrome}

During 6-month follow-up period, the mean AST and ALT levels 
decreased in both control and vitamin E groups. However, the decreases were significantly greater in the vitamin $\mathrm{E}$ group than in the control group: AST decreased by 11 and $22 \mathrm{IU} / \mathrm{L}$ in control and vitamin $\mathrm{E}$ group, respectively $(P<0.05)$, and ALT decreased by 19 and $59 \mathrm{IU} / \mathrm{L}$ in control and vitamin $\mathrm{E}$ group, respectively $(P<0.01)$.(Fig. 2A).

In vitamin E group, the ALT levels were decreased to $46 \pm 34$ IU/ $\mathrm{mL}$ and the AST levels were decreased to $38 \pm 17 \mathrm{IU} / \mathrm{L}$ after 6 month. In control group, the ALT levels were decreased to $83 \pm 54$ $\mathrm{IU} / \mathrm{L}$ and the AST levels were decreased to $51 \pm 23 \mathrm{IU} / \mathrm{L}$ after 6 month.

The number of patients with ALT levels less than $40 \mathrm{IU} / \mathrm{L}$ were $9(16.1 \%)$ and $36(62.1 \%)$ in control and vitamin $\mathrm{E}$ group respectively $(P<0.01)$. The number of patients with AST levels less than $40 \mathrm{IU} / \mathrm{L}$ were $23(41.1 \%)$ and $43(74.1 \%)$ in control and vitamin $\mathrm{E}$ group respectively $(P<0.01)$.

The proportion of patients with ALT reduction $\geq 30 \%$ at 6

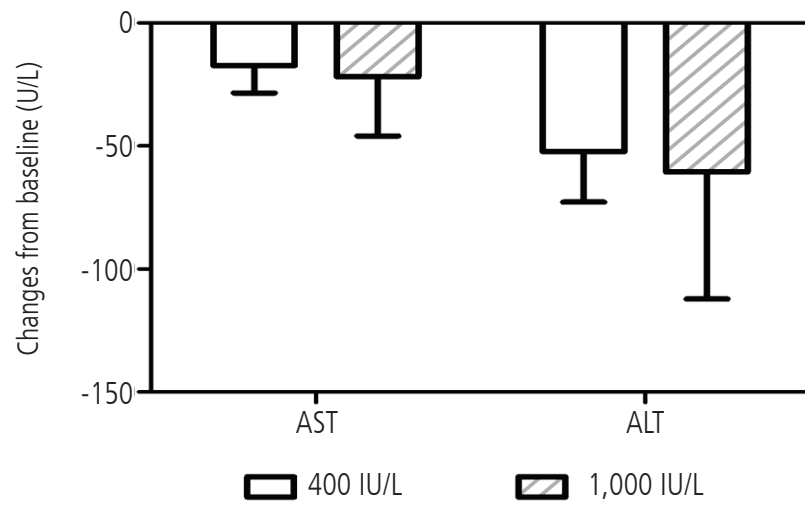

Figure 3. Changes of serum aminotransferase levels according to vitamin E treatment dose in NAFLD patients with metabolic syndrome. Mean values and standard errors of mean are shown for changes in AST and ALT levels over 6 months of vitamin E treatment ( $400 \mathrm{IU}$ and 1,000 IU). NAFLD, nonalcoholic fatty liver disease; AST, aspartate transaminase; ALT, alanine transaminase. months was higher with vitamin $\mathrm{E}$ group than with control group (71.4\% vs. $28.6 \%$, respectively; $P<0.01)$.

When vitamin $E$ was discontinued, the mean ALT level elevated significantly (Fig. 2B) and 54\% of patients had re-elevation of ALT levels by at least $50 \%$ despite continued life style modification.

\section{The changes of ALT and AST levels according to vitamin $\mathrm{E}$ daily dose.}

Among 58 patients taking vitamin E treatment in NAFLD patients with metabolic syndrome, 8 patients $(13.8 \%)$ were taking $400 \mathrm{IU} /$ day, 50 patients (86.2\%) were taking 1,000 IU/day. AST decreased by 17 and $21 \mathrm{IU} / \mathrm{L}$ in $400 \mathrm{IU} /$ day and 1,000 IU/day vitamin E group, respectively, and ALT decreased by 52 and $60 \mathrm{IU} / \mathrm{L}$ in $400 \mathrm{IU} /$ day and 1,000 IU/day vitamin E group, respectively. The ALT and AST levels were decreased in both group compared to baseline but the changes of ALT and AST levels according to vitamin E daily dosage were not different statistically (Fig. 3).

\section{Effects of vitamin E on metabolic profiles of NAFLD patients with metabolic syndrome}

Next, we sought to find out whether vitamin E treatment improves metabolic profiles in NAFLD patients with metabolic syndrome. During the 6-month treatment period, vitamin E did not induce significant changes in body weight, fasting serum glucose or lipid levels compared to control (Table 2).

\section{Safety of vitamin E treatment in NAFLD patients with metabolic syndrome}

In the 82 patients treated with vitamin $E$, no serious complications were observed during vitamin E treatment. Urinary symptoms and prostatic-specific antigen serum levels were regularly

Table 2. Changes in body weight and metabolic profiles during 6-month vitamin E treatment in NAFLD patients with metabolic syndrome

\begin{tabular}{lccc}
\hline & \multicolumn{1}{c}{ Changes from baseline } & \multirow{2}{*}{$\boldsymbol{P}$-value } \\
\cline { 2 - 3 } & Control & Vitamin E & 0.71 \\
\hline Body mass index & $-0.3(-0.7 \sim 0.2)$ & $-0.3(-0.6 \sim 0.1)$ & 0.28 \\
Fasting serum glucose $(\mathrm{mg} / \mathrm{dL})$ & $-18.8(-44.3 \sim 6.8)$ & $-4.3(-14.4 \sim 5.7)$ & 0.79 \\
Triglycerides $(\mathrm{mg} / \mathrm{dL})$ & $22.1(-15.9 \sim 60.1)$ & $30.7(-22.2 \sim 83.6)$ & 0.07 \\
Low-density lipoprotein $(\mathrm{mg} / \mathrm{dL})$ & $-10.9(-22.1 \sim 0.4)$ & $1.9(-6.7 \sim 10.6)$ & 0.17 \\
High-density lipoprotein $(\mathrm{mg} / \mathrm{dL})$ & $-1.9(-5.4 \sim 1.6)$ & $0.9(-1.2 \sim 3.1)$ &
\end{tabular}

Values are represented as the mean (95\% confidence interval).

NAFLD, nonalcoholic fatty liver disease. 
checked during the mean follow-up of 37 months from initiation of vitamin $E$, but the changes of prostatic specific antigen serum levels were no significant differences during vitamin $\mathrm{E}$ treatment.

\section{DISCUSSION}

Weight reduction by life style modification is the main therapeutic option in NAFLD. In order to reduce hepatic inflammation, however, at least $7-9 \%$ of weight reduction is required ${ }_{1}^{28,29}$ but only less than half of patients achieves this goal despite dietary counseling and exercise recommendations. ${ }^{30}$ Therefore, additional therapeutic strategy should be developed for the optimal management of hepatic inflammation in NAFLD. Vitamin E significantly reduces ALT levels and histologic activity scores in biopsy-proven NASH patients, ${ }^{12}$ but currently vitamin $\mathrm{E}$ is not indicated in NAFLD patients without histologic confirmation of inflammation. Since comorbid metabolic syndrome in NAFLD strongly predicts presence of $\mathrm{NASH}_{1}{ }^{15-17}$ it is mechanistically probable that NAFLD patients with metabolic syndrome might benefit from vitamin $E$. However, there have been limited data on the effect of vitamin $E$ in NAFLD. ${ }^{31,32}$

In this retrospective cohort study, we have shown that vitamin $\mathrm{E}$ treatment was significantly associated with improved transaminase levels in NAFLD patients with metabolic syndrome compared to PS-matched control. Since all study patients had been advised to lose weight for at least 6 months before enrollment, our data indicates that vitamin $\mathrm{E}$, in addition to life style modification, promotes improvements of hypertransaminasemia in NAFLD patients with metabolic syndrome.

The mean HOMA-IR values were $>4.65$ in our study population, indicating presence of significant insulin resistance..$^{33}$ Insulin resistance is not only the main basis of abnormal hepatic lipid metabolism in metabolic syndrome ${ }^{30}$ but also a key component in the pathogenesis of steatohepatitis. ${ }^{15,34,35}$ Therefore, our patients with metabolic syndrome have increased probability of having steatohepatitis. ${ }^{7,15-17,34}$ Because both insulin resistance and steatohepatitis is closely related to oxidative stress, ${ }^{30,36-38}$ vitamin $E$ is believed to reduce ALT levels by inhibiting lipid peroxidation and subsequent oxidative stress in our NAFLD patients with metabolic syndrome. ${ }^{39}$

It is an interesting finding that vitamin $\mathrm{E}$ had no effect on metabolic profiles in our patients. Our finding suggests that hepatic steatosis may not be improved by vitamin $\mathrm{E}$. This hypothesis is mechanistically relevant because vitamin E may act on down- stream of hepatic fatty accumulation. Our results also agree with the findings of the PIVENS ${ }^{12}$ and TONIC trials, ${ }^{31}$ and explain the ALT rebound following withdrawal of vitamin $\mathrm{E}$ in our patients.

The main limitation of this study is that only short-term effects of vitamin $E$ were assessed. Therefore, the long-term efficacy and safety of vitamin $E$ in NAFLD patients with metabolic syndrome remain unknown, and prolonged follow-up studies are needed to assess the effects of vitamin $E$ therapy on the morbidity and mortality of NAFLD patients with metabolic syndrome. Secondly, liver biopsy was performed only in a limited number of study patients, so that the baseline inflammatory activity and fibrosis stage were not known. However, the mean NAFLD fibrosis scores were < -1.455 , suggesting that advanced fibrosis may be excluded in our study population. ${ }^{26}$ The main focus of this study was to assess the effect of vitamin $E$ in a NAFLD population without knowledge of liver histology. Of course, NAFLD with metabolic syndrome is not completely consistant with NASH but metabolic syndrome is known to a major risk factor in NAFLD patients and liver biopsy always contains limitations such as high cost and complication. So to try a liver biopsy in all NAFLD patients is difficult in real clinical practice. Our results suggest that vitamin E might be considered in NAFLD patients for the purpose of improving hypertransaminasemia without performing liver biopsy if metabolic syndrome is present. Thirdly, patients in this study were taken some kinds of hepatotonic agents and some of them were taken Pioglitazone. Although the proportion of patients taking hepatotonics or pioglitazone was not differtent between two groups statistically, we need more controlled study because drug interactions were not well known. Fourth, this is a retrospective cohort analysis, so that selection bias might be involved in the results of vitamin $\mathrm{E}$ effect compared to control NAFLD patients. In order to minimize the selection biases, we enrolled the entire NAFLD patient population using EMR-based data warehouse, and PS matching was used to match baseline characteristics that might influence the transaminase levels of study population. As shown in Table 1, vitamin E group showed higher BMI and transaminase levels, but these parameters became comparable after successful PS matching. Finally, the present study evaluated the effect of vitamin $E$ on a surrogate marker (i.e., ALT level) only, and the effects of vitamin E on the histology of NAFLD remained unexplored. A reassessment of the PIVENS trial shows that ALT responses significantly correlated with improvement of histologic features in patients with NASH. ${ }^{13}$ Thus, it is possible that vitamin E may not only reduce ALT, but also improves histologic findings in NAFLD patients with metabolic syndrome. However, the hypothesis that vitamin E-induced ALT 
response leads to improvement of histology and the long-term prognosis in this population need to be proved in the further prospective trials.

In conclusion, short-term vitamin E treatment significantly improves abnormal liver enzyme levels in NAFLD patients with metabolic syndrome, but the metabolic profiles are not affected by vitamin E.

\section{Acknowledgements}

This work was supported by the National Research Foundation of Korea (NRF) and grant funded by the Korean Government (NRF2013R1A1A2061509) to J-W Kim.

\section{Conflicts of Interest}

The authors have no conflicts to disclose.

\section{REFERENCES}

1. Lazo M, Clark JM. The epidemiology of nonalcoholic fatty liver disease: a global perspective. Semin Liver Dis 2008;28:339-350.

2. Vernon G, Baranova A, Younossi ZM. Systematic review: the epidemiology and natural history of non-alcoholic fatty liver disease and non-alcoholic steatohepatitis in adults. Aliment Pharmacol Ther 2011;34:274-285.

3. Ekstedt M, Franzen LE, Mathiesen UL, Thorelius L, Holmqvist M, Bodemar $G$, et al. Long-term follow-up of patients with NAFLD and elevated liver enzymes. Hepatology 2006;44:865-873.

4. Wong VW, Wong GL, Choi PC, Chan AW, Li MK, Chan HY, et al. Disease progression of non-alcoholic fatty liver disease: a prospective study with paired liver biopsies at 3 years. Gut 2010;59:969-974.

5. Adams LA, Lymp JF, St Sauver J, Sanderson SO, Lindor KD, Feldstein $A$, et al. The natural history of nonalcoholic fatty liver disease: a population-based cohort study. Gastroenterology 2005;129:113121.

6. Dunn W, Xu R, Wingard DL, Rogers C, Angulo P, Younossi ZM, et al. Suspected nonalcoholic fatty liver disease and mortality risk in a population-based cohort study. Am J Gastroenterol 2008;103:22632271.

7. Chalasani N, Younossi Z, Lavine JE, Diehl AM, Brunt EM, Cusi K, et al. The diagnosis and management of non-alcoholic fatty liver disease: practice Guideline by the American Association for the Study of Liver Diseases, American College of Gastroenterology, and the American Gastroenterological Association. Hepatology 2012;55:2005-2023.

8. Browning JD, Horton JD. Molecular mediators of hepatic steatosis and liver injury. J Clin Invest 2004;114:147-152.
9. Chang CY, Argo CK, Al-Osaimi AM, Caldwell SH. Therapy of NAFLD: antioxidants and cytoprotective agents. J Clin Gastroenterol 2006;40(Suppl 1):\$51-S60.

10. Hickman I, Macdonald G. Is vitamin E beneficial in chronic liver disease? Hepatology 2007:46:288-290.

11. Pacana T, Sanyal AJ. Vitamin E and nonalcoholic fatty liver disease. Curr Opin Clin Nutr Metab Care 2012;15:641-648.

12. Sanyal AJ, Chalasani N, Kowdley KV, McCullough A, Diehl AM, Bass $N M$, et al. Pioglitazone, vitamin $E$, or placebo for nonalcoholic steatohepatitis. N Engl J Med 2010;362:1675-1685.

13. Hoofnagle JH, Van Natta ML, Kleiner DE, Clark JM, Kowdley KV, Loomba $R$, et al. Vitamin $E$ and changes in serum alanine aminotransferase levels in patients with non-alcoholic steatohepatitis. Aliment Pharmacol Ther 2013;38:134-143.

14. Korean Association for the Study of the Liver (KASL). KASL clinical practice guidelines: management of nonalcoholic fatty liver disease. Clin Mol Hepatol 2013;19:325-348.

15. Marchesini G, Bugianesi E, Forlani G, Cerrelli F, Lenzi M, Manini R, et al. Nonalcoholic fatty liver, steatohepatitis, and the metabolic syndrome. Hepatology 2003;37:917-923.

16. Dixon JB, Bhathal PS, O'Brien PE. Nonalcoholic fatty liver disease: predictors of nonalcoholic steatohepatitis and liver fibrosis in the severely obese. Gastroenterology 2001;121:91-100.

17. Kang H, Greenson JK, Omo JT, Chao C, Peterman D, Anderson L, et al. Metabolic syndrome is associated with greater histologic severity, higher carbohydrate, and lower fat diet in patients with NAFLD. Am J Gastroenterol 2006;101:2247-2253.

18. D'Agostino RB. Propensity score methods for bias reduction in the comparison of a treatment to a non-randomized control group. Statistics in Medicine 1998;17:2265-2281.

19. Hosaka T, Suzuki F, Kobayashi M, Seko Y, Kawamura Y, Sezaki H, et al. Long-term entecavir treatment reduces hepatocellular carcinoma incidence in patients with hepatitis $B$ virus infection. Hepatology 2013;58:98-107.

20. Lim YS, Han S, Heo NY, Shim JH, Lee HC, Suh DJ. Mortality, liver transplantation, and hepatocellular carcinoma among patients with chronic hepatitis B treated with entecavir vs lamivudine. Gastroenterology 2014;147:152-161.

21. Yoo S, Lee KH, Lee HJ, Ha K, Lim C, Chin HJ, et al. Seoul National University Bundang Hospital's Electronic System for Total Care. Healthc Inform Res 2012;18:145-152.

22. Chalasani N, Younossi Z, Lavine JE, Diehl AM, Brunt EM, Cusi K, et al. The diagnosis and management of non-alcoholic fatty liver disease: practice guideline by the American Gastroenterological Association, American Association for the Study of Liver Diseases, and American College of Gastroenterology. Gastroenterology 2012;142:1592-1609.

23. Grundy SM, Brewer HB Jr, Cleeman JI, Smith SC Jr, Lenfant C. Defini- 
tion of metabolic syndrome: Report of the National Heart, Lung, and Blood Institute/American Heart Association conference on scientific issues related to definition. Circulation 2004;109:433-438.

24. Alberti KG, Zimmet P, Shaw J. Metabolic syndrome--a new worldwide definition. A Consensus Statement from the International Diabetes Federation. Diabet Med 2006;23:469-480.

25. Farrell GC, Chitturi S, Lau GK, Sollano JD. Guidelines for the assessment and management of non-alcoholic fatty liver disease in the Asia-Pacific region: executive summary. J Gastroenterol Hepatol 2007;22:775-777.

26. Angulo P, Hui JM, Marchesini G, Bugianesi E, George J, Farrell GC, et al. The NAFLD fibrosis score: a noninvasive system that identifies liver fibrosis in patients with NAFLD. Hepatology 2007;45:846-854.

27. Lin ZH, Xin YN, Dong QJ, Wang Q, Jiang XJ, Zhan SH, et al. Performance of the aspartate aminotransferase-to-platelet ratio index for the staging of hepatitis C-related fibrosis: an updated meta-analysis. Hepatology 2011;53:726-736.

28. Harrison SA, Fecht W, Brunt EM, Neuschwander-Tetri BA. Orlistat for overweight subjects with nonalcoholic steatohepatitis: A randomized, prospective trial. Hepatology 2009;49:80-86.

29. Promrat K, Kleiner DE, Niemeier HM, Jackvony E, Kearns M, Wands $J R$, et al. Randomized controlled trial testing the effects of weight loss on nonalcoholic steatohepatitis. Hepatology 2010;51:121-129.

30. Torres DM, Williams CD, Harrison SA. Features, diagnosis, and treatment of nonalcoholic fatty liver disease. Clin Gastroenterol Hepatol 2012;10:837-858.

31. Lavine JE, Schwimmer JB, Van Natta ML, Molleston JP, Murray KF, Rosenthal $P$, et al. Effect of vitamin $E$ or metformin for treatment of nonalcoholic fatty liver disease in children and adolescents: the TONIC randomized controlled trial. JAMA 2011;305:1659-1668.
32. Polyzos SA, Kountouras J, Zafeiriadou E, Patsiaoura K, Katsiki E, Deretzi $G$, et al. Effect of spironolactone and vitamin $E$ on serum metabolic parameters and insulin resistance in patients with nonalcoholic fatty liver disease. J Renin Angiotensin Aldosterone Syst 2011;12:498-503.

33. Stern SE, Williams K, Ferrannini E, DeFronzo RA, Bogardus C, Stern MP. Identification of individuals with insulin resistance using routine clinical measurements. Diabetes 2005;54:333-339.

34. Chitturi S, Abeygunasekera S, Farrell GC, Holmes-Walker J, Hui JM, Fung $\mathrm{C}$, et al. NASH and insulin resistance: insulin hypersecretion and specific association with the insulin resistance syndrome. Hepatology 2002;35:373-379.

35. Pagano G, Pacini G, Musso G, Gambino R, Mecca F, Depetris N, et al. Nonalcoholic steatohepatitis, insulin resistance, and metabolic syndrome: further evidence for an etiologic association. Hepatology 2002:35:367-372.

36. Evans JL, Maddux BA, Goldfine ID. The molecular basis for oxidative stress-induced insulin resistance. Antioxid Redox Signal 2005;7:1040-1052.

37. Videla LA, Rodrigo R, Araya J, Poniachik J. Insulin resistance and oxidative stress interdependency in non-alcoholic fatty liver disease. Trends Mol Med 2006;12:555-558.

38. Park K, Gross M, Lee DH, Holvoet P, Himes JH, Shikany JM, et al. Oxidative stress and insulin resistance: the coronary artery risk development in young adults study. Diabetes Care 2009;32:13021307.

39. Phung N, Pera N, Farrell G, Leclercq I, Hou JY, George J. Pro-oxidant-mediated hepatic fibrosis and effects of antioxidant intervention in murine dietary steatohepatitis. Int J Mol Med 2009;24:171180. 